\title{
ACUTE SPINAL EPIDURAL ABSCESS : A CASE IN AN INFANT WITH RECOVERY
}

\author{
BY \\ R. G. RUSHWORTH and P. B. MARTIN \\ From Guy's-Maudsley Neurosurgical Unit, and the Belgrave Hospital for Children, London
}

(RECEIVED FOR PUBLICATION OCTOBER 26, 1957)

Acute spinal epidural abscess is a neurosurgical emergency in which early diagnosis and prompt treatment are essential if permanent cord damage is to be avoided. For this reason, this uncommon condition has become well-recognized in recent years, and many full accounts have appeared. However, most of the patients described have been adults, and the following case is reported to illustrate the difficulties of diagnosis in a baby before the appearance of neurological signs.

\section{Case Report}

P.S. was born on April 11, 1955, and was three weeks overdue. The birth weight was $8 \mathrm{lb} .8 \mathrm{oz}$. and the delivery was normal. There was some neonatal skin sepsis, presumably staphylococcal in origin. At the age of 11 weeks he was admitted to the Belgrave Hospital for Children with a pyrexial illness, and a disinclination to move the left arm which was held in Erb's position. No other physical signs were found on admission, and he moved the arm normally after 24 hours. The pyrexia later proved to be due to a staphylococcal pneumonia and empyema, and after a desperate illness he recovered completely.

On July 16, 1956, he was again seen at the Belgrave Hospital, the mother complaining that he had seemed unwell for about two days. He had felt hot, and had screamed whenever he was lifted with her hands beneath his armpits or placed on his back. The feeds had been taken well, there had been no vomiting, and the bowels had been open normally. In February and May, 1956, he had two attacks of fever and irritability each lasting about two days.

On admission the temperature was $100 \cdot 6^{\circ} \mathrm{F}$., pulse 120 and respirations 25 per minute. The child was fretful and did not seem to be in any great pain while lying quietly, but he often screamed when picked up. No localized tenderness was detected to account for this. He was teething and the throat was mildly injected. The cardiovascular and respiratory systems were normal. There was no neck stiffness, and there were no abnormal neurological signs. The spleen was slightly enlarged, but apart from this the abdomen was normal.

Laboratory investigations were carried out: W.B.C. was $7,600 / \mathrm{c}$. mm. (neutrophils $40 \%$ ), film was normal; E.S.R. was $20 \mathrm{~mm}$. in one hour; a patch test and throat swab were negative; radiographs of the spine, chest, pelvis and abdomen were all normal.

The day after admission his condition was unchanged. The next evening his temperature had risen to $103^{\circ} \mathrm{F}$. and the respirations had become more rapid. At 3 a.m. on July 19 he began to twitch and appeared about to convulse, and lumbar puncture was performed that morning. The fluid was clear, but the pressure was not recorded as it fluctuated with the child's crying. The fluid showed: W.B.C. $140 / \mathrm{c}$. mm. (91\% polymorphs), R.B.C. $30 /$ c. mm., protein $300 \mathrm{mg}$. \%, sugar $83 \mathrm{mg}$. \%. A blood-culture taken at this time later grew Staphylococcus aureus.

It was clear that the child was suffering from an infective process, and despite the absence of focal signs it was considered essential to exclude a cerebral abscess. The child was therefore transferred to the Guy's-Maudsley Neurosurgical Unit. On admission there on July 19 he was irritable and crying, and he looked very ill. The temperature was $102 \cdot 4^{\circ} \mathrm{F}$., pulse 134 and respiratory rate 34 per minute. There were no localizing signs of infection and no abnormal neurological signs. He cried when touched or disturbed, but this was not more noticeable when the back was examined. He resented neck flexion, but there was no neck stiffness, and Kernig's sign was not present.

Ventriculography was performed which excluded a cerebral abscess or other space-occupying lesion. Preliminary lumbar puncture gave clear fluid under a pressure of $30 \mathrm{~mm}$., and specimens of cerebrospinal fluid were taken for examination from the right ventricle and from the cerebral subarachnoid space. The results were: lumbar, protein $350 \mathrm{mg}$. \%, W.B.C. 90/c.mm.; right ventricle, protein $55 \mathrm{mg} . \%$, W.B.C. $5 /$ c.mm. As these figures clearly indicated a cerebrospinal fluid block, cisternal puncture was carried out and $2 \mathrm{ml}$. of Ethiodan (ethyl-p-iodophenylundecoate (B.D.H.)) introduced. This was found to be held up largely at the foramen magnum, but it ran in a thin stream down as far as the seventh cervical vertebra, where the obstruction was complete (Fig. 1). To determine the lower limit of the block, a further $2 \mathrm{ml}$. of Ethiodan were introduced by lumbar puncture. This ran up as far as the fifth thoracic vertebra, at which level there was complete obstruction 
(Fig. 2). The appearances were those of an extradural lesion. During the screening procedure the child lay face downward, and he seemed to find this posture particularly uncomfortable. He cried and struggled to turn over. collection of pus was found. It lay mainly to the left, and had pushed the dura to the right. Further pus was found at T1, which proved to be the upper limit. The laminectomy was continued up to include C7 and C6.

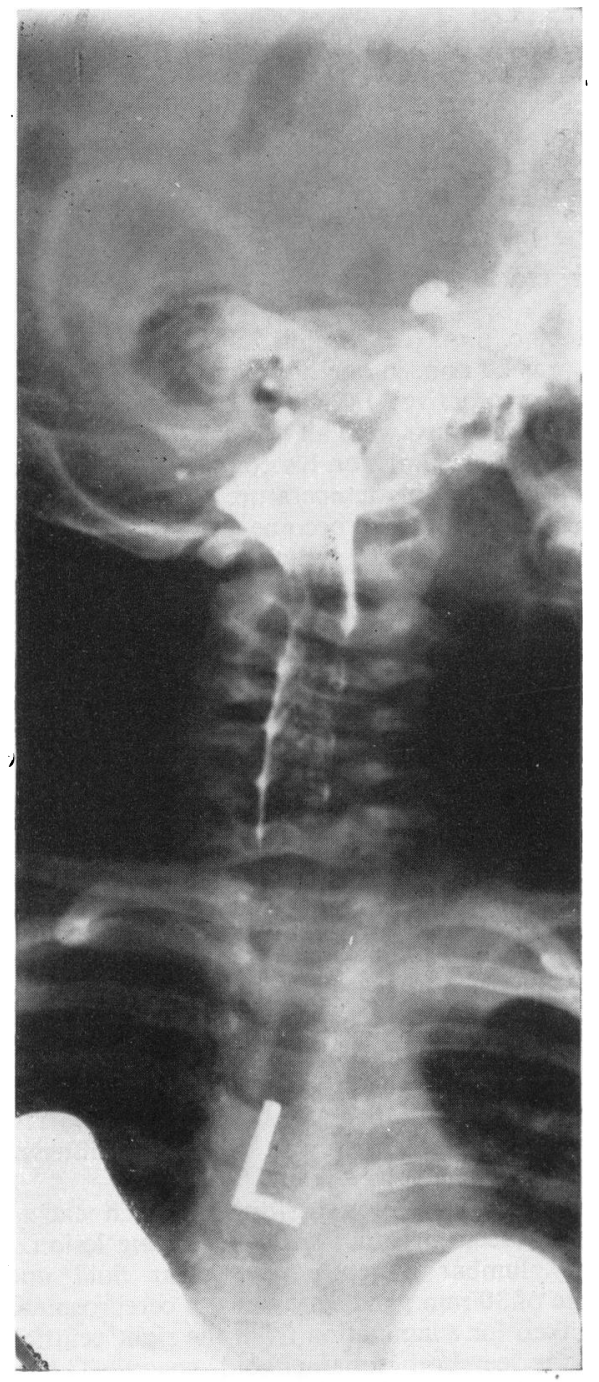

FIG. 1.

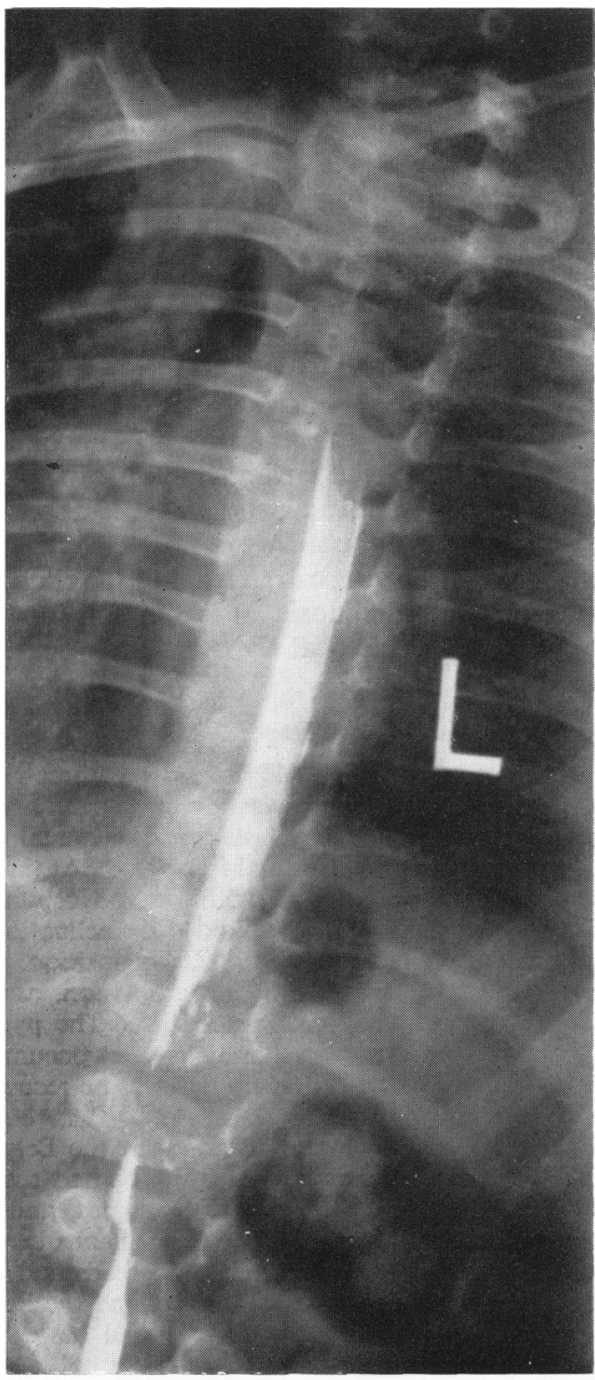

FIG. 2.

Myelographic appearances. In Fig. 1, contrast medium introduced'by the cisternal route is seen to be held up for the most part at the foramen magnum, a little running down in a thin stream as far as the seventh cervical vertebra. In Fig. 2, the flow of the lumbar contrast medium is completely blocked at the fifth thoracic vertebra.

A history of repeated staphylococcal infections and the present pyrexial illness suggested epidural abscess as the most likely cause of the block. Laminectomy was therefore performed immediately. The spines and laminae of the third and fourth thoracic vertebrae were removed without any abnormality being encountered, but when the removal was carried up to T2 a large
A drainage tube was inserted and the wound closed.

Penicillin and streptomycin had been given before operation, and these antibiotics were continued pending sensitivity tests. The organism was identified as a coagulase-positive staphylococcus which was resistant to penicillin, and this was accordingly changed to terra- 
mycin administered systemically, and chloramphenicol instilled locally via the tube.

After some initial anxiety, when the child's temperature rose to $104^{\circ} \mathrm{F}$., recovery was rapid and the wound healed perfectly. No neurological signs appeared in the postoperative period, and he began to walk while in hospital. It was thought advisable to continue systemic antibiotic treatment for four weeks, and he was discharged six weeks after the operation. He has remained perfectly well to date (15 months).

\section{Discussion}

Infection of the spinal epidural space is always secondary to infection elsewhere in the body, either adjacent or remote. The mode of involvement of the space is obvious enough when the focus is near the involved area, such as an infected rib, but more difficult to understand when spread occurs from some distant site. The metastatic pathway must be either lymphatic or haematogenous, the organism lodging in the epidural tissue and giving rise to an abscess. This child met the staphylococcus very early in life, and it must be presumed that between the repeated episodes it lay dormant somewhere in the body.

Staphylococcus aureus has been the organism responsible for acute spinal epidural abscess in almost all reported cases (Heusner, 1948; Hulme and Dott, 1954). According to Neale (1936) this organism possesses a special affinity for the metastatic invasion of loose fatty areolar tissue of the type which fills the epidural space. The site of the invasion depends upon anatomical considerations. Dandy (1926) pointed out that the spinal epidural space was only present dorsal to the spinal nerve attachments, the dura anteriorly being in contact with the vertebral bodies and their ligaments. In the cervical region the space is only a potential one as far down as the seventh vertebra, but then it begins to deepen and is $0.5-0.75 \mathrm{~cm}$. in depth between the fourth and eighth thoracic vertebrae. It becomes shallow again from the eleventh thoracic to the second lumbar vertebra, and thereafter attains its greatest depth. Consequently an epidural abscess is rarely found above the fourth thoracic vertebra, and is commonest in the lower thoracic and lumbar regions. Dandy considered that local trauma was an important inciting agent in the development of haematogenous infection. An alternative view of the mechanism of invasion of the epidural space was put forward by Browder and Meyers (1937). They considered that haematogenous spread first occurred to a vertebra, the epidural space then being secondarily involved from this focus of infection. No evidence of vertebral involvement was seen in the present case, either in the $x$-rays or at operation, but it could have been so small as to be invisible. Once the abscess is established, the spinal cord is in grave danger. Not only is it subjected to direct compression but the infection may also bring about extensive damage by producing thrombosis of its bloodvessels. In the latter case, the most severe neurological signs may occur very suddenly indeed.

The early symptoms in adults are remarkably uniform. There is a pyrexial illness, localized back pain of the most agonizing intensity, and radicular pain. Familiarity with this picture should make the diagnosis possible before neurological signs appear, but, in a young child with a pyrexial illness and no neurological signs, the condition might well be overlooked if attention is not drawn to the back. Although in this case no local tenderness was found, it was thought in retrospect that significance should be attached to the mother's observations on the type of handling which caused him to scream, and to our own observation of his discomfort when he lay face downwards or was picked up. The features which are of diagnostic importance may then be briefly summarized: (1) A history of infection which may have been weeks or months beforehand. Boharas and Koskoff (1941) found the average interval in 85 cases to be 4.2 weeks, but in one case it was as long as three years. (2) Trauma to the back. (3) An unexplained pyrexial illness. (4) Tenderness of the spine. This may be present on physical examination, or may be suggested by the child's disinclination to lie on its back or face downwards.

If an epidural abscess is suspected, lumbar puncture should be performed with caution, and with frequent removal of the stilette and aspiration as the needle is advanced. Pus in the epidural space may be encountered giving the diagnosis, but if the needle is inadvertently passed through the abscess into the subarachnoid space, then meningitis may result. When the subarachnoid space is tapped, the likely finding is a partial or complete spinal block, with the characteristic manometry and protein elevation. There is a moderate pleocytosis.

There are few reports in the literature of myelography as a diagnostic aid. In adults, although the site of the abscess may be determinable by clinical testing, it seems reasonable to perform this investigation to ascertain its exact level and extent. It should be noted though, that lumbar myelography may prove difficult if a second lumbar puncture has to be done to introduce the contrast medium. The initial diagnostic puncture lowers the pressure of the cerebrospinal fluid, which will already be low if spinal block is present, and when a needle is 
introduced for the second time an unsatisfactory flow of the fluid is obtained. In these circumstances the contrast medium is very likely to go between the arachnoid and the dura instead of into the subarachnoid space. It was these considerations that underlay the selection of the cisternal route for introduction. Lumbar myelography then proved to be necessary in addition, and was fortunately successful. In the case of P.S., the apparent block was much longer than the extent of the abscess, which may have been due to an associated arachnoiditis.

The treatment is immediate laminectomy and evacuation of the abscess, a small tube drain being inserted to the site before closing. There is no need to leave the wound open. The appropriate antibiotic should be given systemically and locally. The results are excellent if operation is performed before the onset of neurological signs (Heusner, 1948). Operation is urgently required at this stage, for, as has been pointed out, cord damage may come on with great rapidity. If signs of this have already appeared, then permanent paralysis or death is almost certain.

\section{Summary}

The case is described of a 15 -month-old baby in whom an acute spinal epidural abscess was successfully treated without residual symptoms. The importance of early diagnosis is emphasized, for, if laminectomy and drainage are performed before the onset of neurological signs, complete recovery is to be expected. Once spinal cord damage has taken place, however, then permanent neurological deficit or death is almost certain. The diagnostic difficulties and pathology of the condition are discussed.

We are indebted for permission to report this case to Dr. Mary Wilmers of the Belgrave Hospital, and to Mr. P. H. Schurr, under whose care the child was admitted to the Guy's-Maudsley Neurosurgical Unit. We would like to thank Mr. Murray A. Falconer, Director of the Unit, for his help and advice in the preparation of this paper, and Dr. R. D. Hoare for assistance with the radiological aspects and for permission to reproduce the myelograms.

References

Boharas, S. and Koskoff, Y. D. (1941). J. Amer. med. Ass., 117, 1085 .

Browder, J. and Meyers, E. (1937). Amer. J. Surg., 37, 4.

Dandy, W. E. (1926). Arch. Surg. (Chicago), 13, 477.

Heusner, A. P. (1948). New Engl. J. Med., 239, 845.

Hulme, A. and Dott, N. M. (1954). Brit. med.J., 1, 64.

Neale, A. V. (1936). Bgham med. Rev., 11, 4. 